\title{
Pavlovian aversive to instrumental appetitive transfer: Evidence for across-reinforcement blocking effects
}

\author{
H. FOWLER, J. H. GOODMAN, and M. L. ZANICH \\ University of Pittsburgh, Pittsburgh, Pennsylvania 15260
}

\begin{abstract}
Rats received Pavlovian aversive (shock) conditioning in which white noise was established for different groups as a CS,+ CSO, or CS-. Then, in an appetitive T-maze discrimination, the CSs were presented contingent upon a designated correct response for which food reinforcement was factorially varied at $0,1,2$, or 4 pellets. Although the CS+ suppressed and the CS- facilitated speed of running in the correct arm at the start of discrimination training, these effects extinguished rapidly and did not interact with reward magnitude. Furthermore, choice learning was faciltated by the CS+ and retarded by the CS - , with these effects being comparable for the 1- to 4-pellet reinforcement conditions, but absent for the 0-pellet condition. These findings are difficult to reconcile with a transfer interpretation positing a general signaling property of the CS and are better interpreted as across-reinforcement blocking effects: By predicting a preferred outcome (safety) comparable to the preferred outcome of food reinforcement, the CS- blocks (retards) the association of reinforcement and the $\mathrm{SD}^{\mathrm{D}}$; conversely, by predicting a nonpreferred (shock) outcome discrepant from the preferred food outcome, the CS+ "counterblocks" (enhances) the association of reinforcement and the $\mathrm{SD}^{\mathrm{D}}$.
\end{abstract}

Prior research on Pavlovian aversive $(A v)$ to instrumental appetitive (Ap) transfer has shown that, in a choice discrimination, a danger signal (AvCS +) contingent upon the food-reinforced response does not interfere with but, instead, facilitates learning; conversely, a safety signal (AvCS-), even though a conditioned reinforcer, retards such learning (Fowler, Fago, Domber, \& Hochhauser, 1973). These results have been interpreted as indicating that Pavlovian conditioning involves a dual-learning process: Based on the correlation between the CS and the US (cf. Rescorla, 1967), the primary associative property of the CS is to signal the presence $(\mathrm{CS}+)$ or absence (CS - ) of the US; in addition, the CS acquires a particular affective value depending upon the nature of the US (i.e., Ap or Av) that is present. Jointly, these two properties enable the CS to function as a signal for the presence or absence of either an affectively positive (e.g., food) or an affectively negative (e.g., shock) event and thereby exert control over specific motor and emotional responses.

If the CS's affective value reflects a relatively transient property which is dependent upon the characteristics of the US presently available, then it is conceivable that such a property will be rapidly extinguished and even transformed to a new value in the presence of a qualitatively different reinforcer,

This study was supported in part by United States Public Health Service Grant MH-24115 to the first author. Requests for reprints should be addressed to Dr. Harry Fowler, Department of Psychology, University of Pittsburgh, Pittsburgh, Pennsylvania 15260. while leaving the CS's original signaling value largely intact. In support of such a transformation, Ghiselli and Fowler (1976) have shown, on the basis of differential speed effects, that AvCSs, when contingent upon a running response in an Ap choice discrimination, will rapidly lose their initial suppressing properties and subsequently function as signals for the presence $(\mathrm{CS}+)$ or absence $(\mathrm{CS}-)$ of the new (Ap) reinforcer. Accordingly, when administered for the reinforced response, the AvCS + (as it is transformed) facilitates choice learning by signaling, i.e., mediating, the presence of the reinforcer, whereas the AvCS - retards learning by signaling the reinforcer's absence (Fowler et al., 1973; Ghiselli \& Fowler, 1976). However, when administered for the nonreinforced response, the AvCS + can retard learning by inappropriately signaling the reinforcer's presence, whereas the AvCS - can facilitate learning by appropriately signaling the reinforcer's absence. These reversed effects have been demonstrated in a recent study by Goodman and Fowler (1976).

The present study investigated the relationship between the CS's transformed signaling effect and the magnitude of the $A p$ reinforcer used in discrimination training. In prior studies of the effect, the Ap reinforcer has been held constant at 2-pellets food reinforcement. Given that the CS's affective value is dependent upon the quality and intensity or magnitude of the reinforcer presently available, its transformation (or "counterconditioning") from an AvCS to a signal for Ap reinforcement should be influenced by the magnitude of the Ap reinforcer 
employed in discrimination learning; accordingly, the strength of the observed transfer effects should vary as a function of reward magnitude. To assess this relationship, white noise was established for different groups of rats as an AvCS +, a CSO, or a $\mathrm{CS}$ - and then was administered in discrimination training contingent upon a designated correct response for which the $A p$ (food) reinforcer was factorially varied at $0,1,2$, or 4 pellets. Inclusion of the 0 -pellet condition was for the purpose of extending the magnitude dimension to its lower limit and of assessing a possible "dynamogenic" effect of the CSs, i.e., a facilitating effect based on the emotional excitement or arousal generated by the CSs themselves.

\section{METHOD}

\section{Subjects}

The subjects were 96 naive male albino rats of the SpragueDawley strain, obtained from the Holtzman Company at 90 100 days of age, weighing $300-350 \mathrm{~g}$. The subjects were caged individually and were maintained on a reversed day-night (12-h) cycle.

\begin{abstract}
Apparatus
The Pavlovian-conditioning apparatus was a black wooden chamber $(27.17 \times 19.55 \times 15.24 \mathrm{~cm})$ with a grid floor of stainless steel rods $(0.24 \mathrm{~cm}$ in diameter) spaced $1.59 \mathrm{~cm}$ apart. The rods were connected through timing circuitry and a $0.24-\mathrm{megohm}$ series resistor to the output of a transformer which provided the US: a $0.2-\mathrm{sec}, 60-\mathrm{Hz}$ ac shock of $120 \mathrm{~V}$, as measured across the transformer output (about $0.5 \mathrm{~mA}$ to the grid). A Jensen P4V3 $10.16-\mathrm{cm} \mathrm{3.2-ohm} \mathrm{speaker,} \mathrm{centered} \mathrm{on} \mathrm{the} \mathrm{clear} \mathrm{acrylic-plastic}$ top of the chamber, was connected through timing circuitry to the output of a Grason-Stadler white-noise generator (Model 901B), which provided a 0.2-sec 70-dB CS. CS intensity was determined against an ambient sound level of 60-65 dB.

The discrimination-training apparatus was an enclosed wooden T-maze of uniform height and width (for details, see Fowler et al., 1973). Guillotine doors at the entrance to each arm prevented retracing; additional doors located $20.3 \mathrm{~cm}$ from the end of the stem and $31.3 \mathrm{~cm}$ from the end of each arm provided the start- and goalboxes. Each goalbox had a floor-recessed food well and a frosted acrylic-plastic end wall. The end walls were visible from the choice point and were differentially illuminated from behind by $10-\mathrm{W}$ incandescent bulbs to provide the discriminative stimuli. To effect a relatively difficult bright-dim discrimination, the bulbs illuminating the end walls were operated at 120 and $62.5 \mathrm{~V} \mathrm{ac}$, yielding 75.0 and $9.0 \mathrm{fc}$ of light, respectively, as measured $2.54 \mathrm{~cm}$ in front of the end wall.

An identical Jensen speaker mounted on the clear acrylicplastic top of each maze arm $6.67 \mathrm{~cm}$ before the goalbox door (at about the midpoint of the arm) was used to deliver the same white-noise $\mathrm{CS}(0.2 \mathrm{sec}, 70 \mathrm{~dB})$ when the subject interrupted an infrared photoelectric beam transecting the arm at a point coincidental with the speaker. Additional photoelectric beams located $6.8 \mathrm{~cm}$ before the goalbox end wall and $24.3 \mathrm{~cm}$ from the center of the choice point in each arm were used to measure running latency. For statistical treatment, the latencies were transformed to speed scores (10/latency in sec).
\end{abstract}

\section{Procedure}

The experimental procedure included pretraining, Pavlovianconditioning and discrimination-training phases. One week prior to pretraining, the subjects were started and maintained on a daily diet of $11 \mathrm{~g}$ of Wayne Lab Blox, with water available ad lib.
(This diet was later adjusted to account for food received in the T-maze.) Pretraining was designed to habituate the subjects to the T-maze and to reduce possible position and brightness preferences. Each subject received a total of 16 forced-choice, foodreinforced trials administered four per day at an intertrial interval (ITI) of about $5 \mathrm{~min}$. Daily forced-choice trials were randomly distributed with the restriction that they be balanced over left and right arms and bright and dim goals. P.J. Noyes Formula A rat pellets $(4 \mathrm{~mm}, 45 \mathrm{mg}$ ) served as food reinforcement and, on the first pretraining day, were liberally spread throughout the goalbox. On subsequent days, the pellets were systematically reduced in number and locus until, on the last pretraining day, only two pellets were available in the goalbox food well.

Pavlovian aversive conditioning began on the day following pretraining. Each subject received a total of 96 discrete, 30-sec trials, administered 16 per day at an ITI of about $10 \mathrm{~min}$. The subjects were randomly assigned in equal number to three training conditions designed to establish either an AvCS,$+ \mathrm{CS}-$, or CS0. For CS + subjects, the CS was administered $2.0 \mathrm{sec}$ prior to a single US presentation at $5-25 \mathrm{sec}$ into the trial on a random half of the daily trials; on the other half of the trials, neither event (i.e., a blank trial) occurred. For CS - subjects, the CS was presented once per trial on a random half of the daily trials and the US was presented once per trial on the other half of the trials, each at times within their respective trials matching their occurrence for CS + subjects. For CSO subjects, half of the daily trials were of the CS + variety (CS $2.0 \mathrm{sec}$ prior to the US and a blank trial) and half were of the CS - variety (CS alone and US alone), yielding a "balanced" control (see Fowler et al., 1973). Over the course of training, these trial schedules generated positive, zero, and negative CS-US correlations, i.e., $\mathrm{p}(\mathrm{US} / \mathrm{CS})-\mathrm{p}(\mathrm{US} / \overline{\mathrm{CS}})=$ $+1.0,0.0$, and -1.0 , for $\mathrm{CS}+, \mathrm{CS} 0$, and $\mathrm{CS}-$ subjects, respectively. However, on the first day of Pavlovian conditioning, the scheduling of CSO trial types produced an initial positive bias which reached a zero probability by Trial 16 .

Appetitive discrimination training in the T-maze began on the day following Pavlovian aversive conditioning. Each subject received four free-choice, noncorrection trials per day for the first 6 days and the eight per day, at an ITI of about $5 \mathrm{~min}$. Subjects of each of the three Pavlovian training conditions (CS +, $\mathrm{CSO}$, and $\mathrm{CS}-$ ) were randomly assigned to four groups of eight subjects each which received $0,1,2$, or 4 pellets of food reinforcement for each response to a designated correct goal. Detention time in the correct, as well as incorrect, goal was held constant at $10 \mathrm{sec}$ for all subjects. The brightness of the correct goal was counterbalanced for subjects within each group and its position varied across trials according to a Gellerman sequence. All subjects received a single $C S$ presentation contingent upon each response to the correct goal. Reinforced ( 1 to 4 pellets) subjects were continued in training until reaching a criterion of 19 correct responses out of 20 consecutive trials with the last 12 correct. Zero-pellet subjects were continued in training until reaching an "extinction" criterion of two consecutive 5-min no-choice trials on which the first photoelectric beam in the maze arm was not interrupted, or until a maximum of 200 trials had been administered. For purposes of statistical treatment, the error score of any 0 -pellet subject having less than 200 trials was adjusted to a 200 -trial baseline, i.e., (errors/actual trials) $\times 200$.

\section{RESULTS}

\section{Learning Measures}

Figure 1 presents mean errors and trials to criterion (left and right panels, respectively) as a function of CS-US correlation for subjects of the $0-, 1$, 2-, and 4-pellet reinforcement conditions. Only error scores are presented for the 0-pellet subjects because of their 200-trial cutoff. As indicated in Figure 1, 
CS-US correlation had no observable effect on the error means for the 0-pellet subjects and yielded a horizontal function. A separate analysis of these data showed no significant CS effect $(F<1)$. Similarly, there was no significant $\mathrm{CS}$ effect $(\mathrm{F}<1)$ on actual (unadjusted) error scores for the 0-pellet subjects, the means for the CS + , CSO, and CS - subgroups being 97.6, 97.0, and 95.8, respectively. In addition, analysis of error scores for the 0-pellet subjects throughout different stages of training showed no significant CS effects.

In comparison, Figure 1 shows that errors to criterion for the reinforced (1- to 4-pellet) subjects were increasingly reduced by larger reward magnitudes, in evidence of learning at progressively faster rates. Furthermore, for the reinforced subjects, errors were reduced by the $\mathrm{CS}+$ and increased by the $\mathrm{CS}$ - relative to the CSO controls, with these effects being common across the reinforcement conditions (except in the 4-pellet case where the CS + effect was constrained, possibly as a result of a "floor" effect). Analysis of these data (excluding the 0-pellet subjects for which the imposed training cutoff produces artifactually low error variance) showed both a significant reward-magnitude effect $[\mathrm{F}(2,54)=6.14, \mathrm{p}<.005]$ and a significant $\mathrm{CS}$ effect $[F(2,54)=4.20, p<.025]$. In addition, trend analyses of these effects by orthogonal polynomial contrasts showed significant linear components which accounted for virtually all $(99.9 \%)$ of the reward-magnitude variance $[\mathrm{F}(1,54)=12.27$, $\mathrm{p}<.001]$ and for most $(85.1 \%)$ of the CS variance $[\mathrm{F}(1,54)=7.17, \mathrm{p}<.01]$; in both cases, residualtrend variance was negligible $(F<1$ and $F=1.25$, respectively). There was no interaction of the rewardmagnitude and $C S$ variables $(F<1)$ or reliable trend components to the interaction data. (An ancillary contrast showed that the difference between the CS +

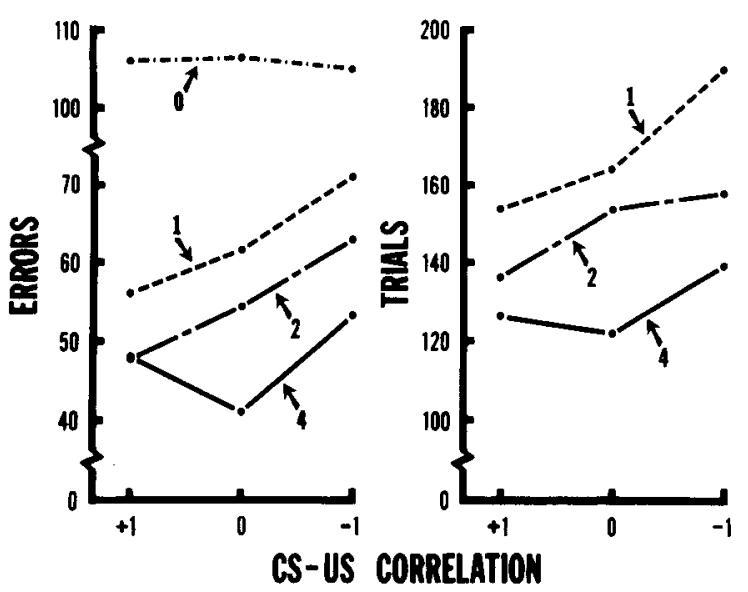

Figure 1. Mean errors and trials to criterion (left and right panels, respectively) as a function of CS-US correlation for subjects of the $0-, 1-, 2-$, and 4-pellet reinforcement groups. (The data for the 0-pellet subjects are restricted to errors because of a 200-trial cutoff.) and CSO groups of the 4-pellet condition was not reliable.) The F-test results did, however, show a significant $\mathrm{S}^{\mathrm{D}}$-brightness effect $[\mathrm{F}(1,54)=28.04$, $\mathrm{p}<.001$ ], indicating slower learning in approaching the bright $\mathrm{S}^{\mathrm{D}}$. This effect did not interact with the other variables.

The right panel of Figure 1 indicates virtually identical effects for the reinforced subjects with a measure of trials to criterion. Like the error data, trials to criterion were linearly related to both reward magnitude and CS-US correlation $[\mathrm{Fs}(1,54)=14.06$ and $4.59, \mathrm{p}<.001$ and $\mathrm{p}<.05$, respectively], with these trend components accounting for virtually all of the variance attributable to the variables $(99.9 \%$ and $95.9 \%$, respectively). Again, there was no interaction of the variables $(F<1)$ or reliable trend components to the interaction data. However, the

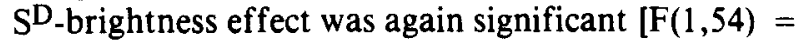
$30.86, p<.001]$.

To determine whether the additive, i.e., noninteractive, relationship between reward magnitude and CS-US correlation for the 1 to 4-pellet subjects was in any way influenced by unit-of-measurement problems (relating to the possibility that different segments of a measurement scale are differentially sensitive to an effect), both the error and trial data for the individual reward-magnitude conditions were transformed to $\mathrm{Z}$ scores. Analyses of these $\mathrm{Z}$ scores showed that there were no reliable interactions or underlying trend components to the interaction data (Fs $<1$ ); however, CS-US correlation effects were still reliable [linear $F s(1,54)=7.24$ and 4.54, $\mathrm{p}<.01$ and $\mathrm{p}<.05$ for errors and trials, respectively; residual Fs $\leqslant 1.08$ ).

\section{Performance Measures}

Analyses of errors and trials either overall or for various stages of training provided no evidence of an aversive or suppressing effect of the AvCS + for either reinforced or nonreinforced (0-pellet) subjects. Although such an effect could be manifested in an avoidance of the CS arm (leading to increased errors and trials), the difficulty of the present choice problems obscures such an effect, as any avoidance conditioned to the $\mathrm{S}^{D}$ can generalize to the similar $S^{\Delta}$. To provide a more sensitive assessment of the aversive properties of the CS, analysis was made of differences in speed of running in the presence of the $\mathrm{CS}$ and in its absence $(\overline{\mathrm{CS}})$ during the initial discrimination trials, immediately following Pavlovian conditioning.

Figure 2 presents mean running speeds over the first five CS and first five $\overline{\mathrm{CS}}$ presentations (left and right panels, respectively) as a function of CS-US correlation for subjects of the 0-, 1-, 2-, and 4-pellet groups. As shown, speeds in both the CS and $\overline{C S}$ arms were positively related to reward magnitude, indicating a general (or generalized) effect of reinforcement during the early discrimination trials. 


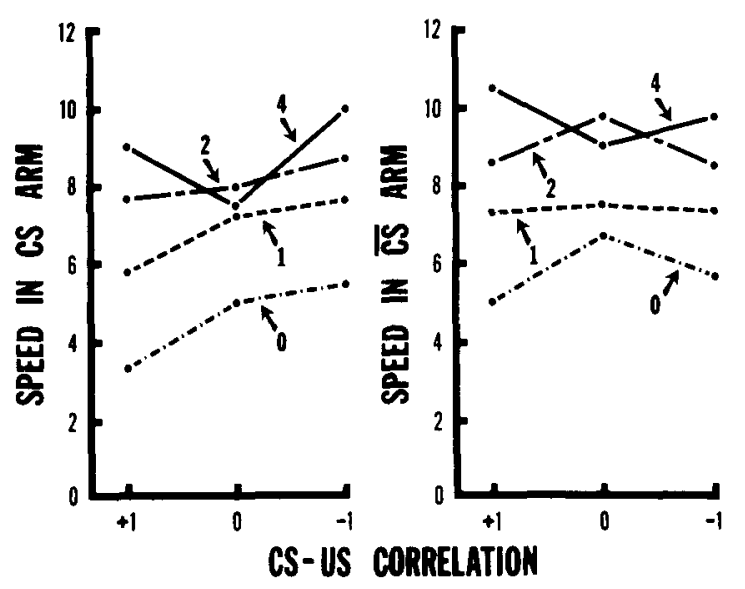

Figure 2. Mean running speeds in the $\mathrm{CS}$ and $\overline{\mathrm{CS}}$ arms (left and right panels, respectively) over the first five trials of each, as a function of CS-US correlation for subjects of the $0-, 1-, 2-$, and 4-pellet reinforcement conditions.

However, only in the CS arm was there any indication that speeds were suppressed for the CS + subjects and facilitated for the $\mathrm{CS}$ - subjects relative to the CSO controls. Analyses of these data showed a significant linear effect of reward magnitude on both CS and $\overline{C S}$ speeds, accounting for $93.8 \%$ and $98.0 \%$ of the variances, respectively $[\mathrm{Fs}(1,27)=$ 23.07 and 26.13 , respectively, ps $<.001$ ]. In addition, the linear effect of CS-US correlation approached significance in the case of CS speeds $[\mathrm{F}(1,72)=3.44, \quad \mathrm{p}<.08]$, but was completely absent for $\overline{\mathrm{CS}}$ speeds $(\mathrm{F}<1)$. All residual-trend effects were negligible (Fs $<1$ ). Similarly, there was no interaction of the $C S$ and reward-magnitude variables $(\mathrm{F}<1)$.

To check on the reliability of the CS effect, the speed data for both CS and $\overline{\mathrm{CS}}$ presentations were combined to form a suppression ratio. This ratio took the familiar form, $\mathrm{A} /(\mathrm{A}+\mathrm{B})$, where $\mathrm{A}$ refers to $C S$ speed and $B$ refers to $\overline{C S}$ speed for individual subjects. Analysis of these data showed a significant linear effect of CS-US correlation $[F(1,72)=$ $5.15, \mathrm{p}<.05]$ and negligible residual-trend variance $(F<1)$, indicating that the CS + suppressed and the CS - facilitated performance. This CS effect did not interact with reward magnitude (the same held true with $\mathrm{Z}$ score transforms), and there was now no main effect of the reward-magnitude variable, indicating that the prior effect for both CS and $\overline{\mathrm{CS}}$ speeds was, in fact, a general effect. Analysis of suppression ratios over the course of additional training trials showed that the CS effect extinguished comparably rapidly for all groups and was completely absent by the third block of five CS and five $\overline{\mathrm{CS}}$ presentations. Furthermore, there was no indication of a reversal of speed effects attributable to the CSs on subsequent trials prior to the onset of discrimination learning.

\section{DISCUSSION}

Comparable to Ghiselli and Fowler's (1976) findings, the present results show that the CSs initially exerted differential suppressing effects on speed of running, attesting to the conditioned aversive property of the $\mathrm{CS}+$ and the conditioned reinforcing property of the CS - relative to the CSO control. These differential speed effects also extinguished rapidly but, surprisingly, they were unaffected by reward magnitude even though this variable itself affected speed of running. Furthermore, unlike Ghiselli and Fowler's findings, there was no indication of a reversal of CS effects on speed performance early in training, as would be expected if the CSs were rapidly transformed to signals for the presence $(\mathrm{CS}+)$ or absence $(\mathrm{CS}-)$ of the new, Ap reinforcer. Although this outcome might be reconciled with Ghiselli and Fowler's findings on the basis of the relatively weak shock-US employed in the present study (their reversal effect was apparent only with slightly stronger USs), the absence of such an effect questions whether Ghiselli and Fowler's finding was a truly independent effect in support of the CS's transformation or a by-product of instrumental learning. Because Ghiselli and Fowler also presented their CSs in the reinforced arm, the faster learning evident in reduced errors and trials for the CS + subjects (and the slower learning evident for the CS - subjects) may have been reflected as well in faster (and slower) speeds for these subjects to the $\mathrm{S}^{\mathrm{D}}$. Hence, it is not clear from their findings that the reversed speed effects were, in fact, the basis for the observed learning (error and trial) differences rather than an additional manifestation of them.

Like the results of prior studies demonstrating positive transfer from Pavlovian Av to instrumental Ap conditioning (e.g., Fowler et al., 1973; Ghiselli \& Fowler, 1976; Goodman \& Fowler, 1976), the present findings show that an AvCS + contingent upon the food-reinforced response facilitated and an AvCS - retarded discrimination learning. In the present study, these effects were limited to the reinforcement (1- to 4-pellet) conditions; there was no evidence of a transfer effect for the nonreinforced (0-pellet) subjects, indicating both that the effect is independent of any "dynamogenic" effect of the CSs and, indeed, is dependent upon the presence of food reinforcement. The fact, however, that the transfer effects for the reinforced subjects were independent of Ap reward magnitude, which itself influenced rate of discrimination learning, seems inconsistent with a signal-affect interpretation of the phenomenon, as one would expect a transformation or "counterconditioning" of the CS's affective value (from Av to Ap) to be modulated by the magnitude of the Ap reinforcer. This disparity cannot be dismissed on the basis of a unit-of-measurement prob- 
lem; the Z-score transforms of the data for the individual reward-magnitude conditions also showed no interactive effect of this variable.

The absence of a CS by reward-magnitude interaction may not be too distressing in view of Ghiselli and Fowler's (1976) finding that the signaling property of the CS is influenced by US-shock intensity, in particular, that AvCSs based on stronger shock USs will, within limits, produce stronger transfer effects. Ghiselli and Fowler's interpretation of this outcome is that, although the CS's signaling property is independent of any affect generated by the US, it is nevertheless influenced by the "impact" or salience of the US over a low range of intensities, because only to the extent that the subject clearly detects the US can it associate presence or absence of the US with the CS. In line with this argument, then, the present findings might be viewed as supporting the assumption that, apart from US salience, the CS's signaling property is independent of US quality and intensity or magnitude, and reflects a binary or nominal $(1,0$; yes, no) processing of information by the subject: Given that the CS's Av affect is rapidly extinguished, the $\mathrm{CS}$ as a signal will provide information about the presence or absence of the new, Ap reinforcer, but not about its magnitude. Still, one is left with the unresolved problem of how the affective value of the new, Ap reinforcer is translated to the CS for, without such a value, the nominal information provided by the CS cannot be of any use in determining the animal's choice or preference behavior.

In view of this difficulty and the asociated problem of establishing independent evidence for the CS's transformation, we believe that it is profitable to consider an alternative interpretation, one that focuses, not on a transfer of the CS's signaling property, but rather on a contrast between what the subject learns to expect in Av conditioning and what it receives as an outcome in Ap discrimination training. In short, we are inclined to argue that the transfer findings are equally, if not more, amenable to a blocking interpretation (e.g., Kamin, 1968, 1969; Rescorla \& Wagner, 1972). Such an interpretation can be applied to the present findings by assuming, quite reasonably, that Ap and Av reinforcers and the expectancies generated by CSs based on these reinforcers are scalable on a common dimension, e.g., a positive-to-negative incentive or a "good-bad" outcome dimension. Accordingly, when AvCSs are presented contingent upon the food-reinforced response, i.e., in the presence of the $S^{D}$, the "good" outcome of food reinforcement should produce little surprise or a relatively small discrepancy as compared with the "good" outcome (safety) predicted by the AvCS - ; hence, the AvCS - should block (retard) the association of food reinforcement and the $\mathrm{S}^{\mathrm{D}}$. Conversely, the AvCS + as a signal for a "bad" outcome (shock) should set the occasion for considerable surprise or a relatively large discrepancy when contrasted with the "good" food outcome and thus it should "counterblock" (enhance) the association of food reinforcement and the $\mathrm{S}^{\mathrm{D}}$ (cf. Rescorla, 1971, Experiment 1; Wagner, 1969b). Relatedly, when the CSs are presented for the nonreinforced response (Goodman \& Fowler, 1976), the AvCS + should block and the AvCS - should counterblock the association of nonreinforcement (a negative-incentive condition) and the $S^{\Delta}$ (cf. Mackintosh \& Turner, 1971; Suiter \& LoLordo, 1971). As a result, the effects of the CSs will be reversed.

A blocking interpretation has no difficulty in assimilating the effects of both reward-magnitude and US-intensity manipulations. As in the present study, the transfer effects for the reinforced subjects will be independent of Ap reward magnitude because the values of the AvCSs $(+, 0$, or -$)$ are set in Pavlovian conditioning and, as constants, they will yield constant differences across $\mathrm{CS}+, \mathrm{CS} 0$, and CS - groups when contrasted with whatever magnitude of food reinforcement is used in discrimination training. (In the vernacular of Rescorla and Wagner's reinforcement-limit theory, lambda will vary as a function of reward magnitude, but the values of the AvCS compounds, being fixed for CS,$+ \mathrm{CS} 0$, and $\mathrm{CS}$ - groups, will yield additive, i.e., noninteractive, differences.) In comparison, the 0-pellet condition cannot provide the basis for a contrast effect and thus, to the extent that the error and trial measures are at all sensitive, they will show only differential suppressing effects of the AvCSs. (As noted, such measures are typically insensitive to suppression effects in a difficult discrimination, and therefore one must rely on a more sensitive, e.g., speed, measure; see Figure 2). Contrastingly, when US-shock intensity is varied in Pavlovian conditioning and reward magnitude is held constant in discrimination training, as in the Ghiselli and Fowler (1976) study, the transfer effects should vary directly with US intensity: Stronger USs will produce stronger conditioning effects and thus more divergent $\mathrm{CS}+, \mathrm{CS} 0$, and CS - values; hence, when contrasted with a constant value (lambda) of food reinforcement, these CSs will generate a wider range of contrast effects, leading (within limits) to greater blocking and counterblocking effects. (A limit is set, for example, by the direct suppressing effect of a responsecontingent AvCS +, i.e., as a conditioned punisher.)

Whether a blocking interpretation will suffice as an explanation of these and similar findings (e.g., Overmier \& Bull, 1970; Overmier \& Payne, 1971) purporting to demonstrate across-reinforcement transfer of the CS's signaling property is obviously 
a matter for future research. Nevertheless, the offered interpretation finds strong support in Konorski and Szwejkowska's (1956) findings, showing that, while it is difficult in Pavlovian conditioning to transform an AvCS + into a ApCS + (or vice versa), it is a relatively easy matter to recondition an AvCS - into a signal for food (or an ApCS - into a signal for shock). These and similar findings obtained by Brown and Wagner (1964; see also Wagner, 1969a) in the context of instrumental conditioning clearly point up the functional equivalence across reinforcement systems of "good" signals (ApCS + and AvCS - ) and of "bad" signals (AvCS + and ApCS - ) and of the opposition of these two sets, as is required by the present interpretation but actually opposed by a signaling interpretation. What would appear necessary, then, to substantiate the present interpretation is a demonstration of acrossreinforcement blocking effects in a more traditional paradigm, e.g., where an $\operatorname{ApCS}(+, 0$, or -$)$ is presented in compound with an added stimulus that is followed by shock in an onbaseline CER procedure.

\section{REFERENCES}

Brown, R. T., \& W AGNER, A. R. Resistance to punishment and extinction following training with shock or nonreinforcement. Journal of Experimental Psychology, 1964, 68, 503-507.

Fowler, H.. Fago, G. C., Domber, E. A., \& Hochhauser, M. Signaling and affective functions in Pavlovian conditioning. Animal Learming \& Behavior, 1973, 1, 81-89.

Ghiselli, W. B., \& Fowler, H. Signaling and affective functions of conditioned aversive stimuli in an appetitive choice discrimination: US intensity effects. Learning and Motivation, 1976, 7, 1-16.

Goodman, J. H., \& Fowler, H. Transfer of the signaling properties of aversive CSs to an instrumental appetitive discrimination. Learning and Motivation, 1976, 7, 446-457.

Kamin, L. J. "Attention-like" processes in classical conditioning. In M. R. Jones (Ed.), Miami symposium on the prediction of behavior: Aversive stimulation. Miami: University of Miami Press, 1968. Pp. 9-31.

Kamin, L. J. Predictability, surprise, attention and conditioning. In B. A. Campbell \& R. M. Church (Eds.), Punishment and aversive behavior. New York: Appleton-Century-Crofts, 1969. Pp. 279.296.

Konorski, J., \& SzWEJKowska, G. Reciprocal transformations of heterogeneous conditioned reflexes. Acta Biologiae Experimentalis, 1956, 17, 141-165.

Mackintosh, N. J., \& TuRner, C. Blocking as a function of novelty of CS and predictability of UCS. Quarterly Journal of Experimental Psychology, 1971, 23, 359-366.

Overmier, J. B., \& Bull, J. A., IIl. Influences of appetitive Pavlovian conditioning upon avoidance behavior. In J. $\mathrm{H}$. Reynierse (Ed.), Current issues in animal learning: $A$ colloquium. Lincoln: University of Nebraska Press, 1970. Pp. 117-141.

Overmier, J. B., \& Payne, R. J. Facilitation of instrumental avoidance learning by prior appetitive Pavlovian conditioning to the cue. Acta Neurobiologiae Experimentalis, 1971, 31, 341-349.

Rescorla, R. A. Pavlovian conditioning and its proper control procedures. Psychological Review, 1967, 74, 71-80.

RESCORLA, R. A. Variation in the effectiveness of reinforcement and nonreinforcement following prior inhibitory conditioning. Learning and Motivation, 1971, 2, 113-123.

Rescorla, R. A., \& WAgNeR, A. R. A theory of Pavlovian conditioning: Variations in the effectiveness of reinforcement and nonreinforcement. In A. H. Black \& W. F. Prokasy (Eds.), Classical conditioning II: Current research and theory. New York: Appleton-Century-Crofts, 1972. Pp. 64-99.

SuITER, R. D., \& LOLORDO, V. M. Blocking of inhibitory Pavlovian conditioning in the conditioned emotional response procedure. Journal of Comparative and Physiological Psychology, 1971, 76, 137-144.

W AGNER, A. R. Frustrative nonreward: A variety of punishment. In B. A. Campbell \& R. M. Church (Eds.), Punishment and aversive behavior. New York: Appleton-Century-Crofts, 1969. Pp. 157-181. (a)

W AGNER, A. R. Stimulus selection and a "modified continuity theory." In G. H. Bower \& J. T. Spence (Eds.), The psychology of learning and motivation (Vol. 3). New York: Academic Press, 1969. Pp. 1-40. (b)

(Received for publication September 14, 1976; revision accepted December $1,1976$. 